\title{
Comparison of Bone Mineral Density Between Male Masters Runners and Cyclists
}

\author{
David G. Lajza*, Aaron C. Tribby, Kaitlyn M. Patterson, Caitlin D. Stover, \\ David A. Geddum, Takashi Abe \\ Faculty: Kaelin C. Young \\ Department of Exercise Science, College of Education
}

For maintenance of bone health during adulthood, weight-bearing exercise is recommended. Cycling is becoming a popular form of exercise for older adults due to reduced impact on weight-bearing joints. Some data suggest that bone mineral density (BMD) is reduced in cyclists due to its low-impact. The purpose of this study was to compare BMD of the spine and proximal femur between 10 male masters runners and 12 cyclists aged 51-76 years using dual energy $\mathrm{x}$-ray absorptiometry. Masters runners had significantly greater BMD of both the total hip $(\mathrm{p}=0.04)$ and femoral neck ( $\mathrm{p}=0.01$ ) sites of the non-dominant leg compared to masters cyclists. Similarly, masters runners tended to have greater BMD of the lumbar spine compared to cyclists, but this difference did not reach statistical significance $(\mathrm{p}=0.053)$. 